Supporting Information for:

\title{
Structural and Electron-Transfer Characteristics of O-, S-, and Se-Tethered Porphyrin Monolayers on Si(100)
}

Amir A. Yasseri, Dennis Syomin, Robert S. Loewe, Jonathan S. Lindsey, Francisco Zaera, and David F. Bocian

Table of Contents:

Experimental section for preparation of $m e s-\mathrm{ZnPCH}_{2} \mathrm{OH}$

Pages

S1-S3

\section{Experimental Section}

An $\mathrm{A}_{3} \mathrm{~B}$-porphyrin bearing a meso-ester group was prepared by mixed-aldehyde condensation of ethyl glyoxylate, mesitaldehyde, and pyrrole using $\mathrm{BF}_{3} \cdot \mathrm{O}(\mathrm{Et})_{2} / \mathrm{EtOH}$ cocatalysis $^{\mathrm{S} 1}$ (Scheme S1). Free base porphyrin 1 was obtained in $2.9 \%$ yield. Subsequent metalation with $\mathrm{Zn}(\mathrm{OAc})_{2} \cdot 2 \mathrm{H}_{2} \mathrm{O}$ afforded $\mathbf{Z n - 1}$ in $92 \%$ yield. Treatment of $\mathbf{Z n - 1}$ with $\mathrm{LiAlH}_{4}$ in THF gave the target porphyrin alcohol mes- $\mathbf{Z n P C H} \mathbf{H}_{2} \mathbf{O H}$ in $34 \%$ yield.

General. All ${ }^{1} \mathrm{H}$ NMR spectra (300 or $\left.400 \mathrm{MHz}\right)$ and ${ }^{13} \mathrm{C}$ NMR $(75 \mathrm{MHz})$ were collected in $\mathrm{CDCl}_{3}$ unless noted otherwise. Absorption and fluorescence spectra were collected in toluene at room temperature. Mass spectra of porphyrins were obtained via laser desorption mass spectrometry (LD-MS) without a matrix, ${ }^{\mathrm{S} 2}$ and by high-resolution fast atom bombardment mass spectrometry (FAB-MS) using a matrix of nitrobenzyl alcohol and polyethylene glycol. Melting points are uncorrected. Silica gel (40 $\mu \mathrm{m}$ average particle size) was used for column chromatography. THF was freshly distilled from sodium as required. Toluene was distilled from $\mathrm{CaH}_{2}$. $\mathrm{CHCl}_{3}$ was stabilized with $0.8 \%$ ethanol.

5-Ethoxycarbonyl-10,15,20-trimesitylporphyrin (1). Following a standard procedure, ${ }^{\mathrm{S} 1}$ a solution of mesitaldehyde $(2.21 \mathrm{~mL}, 15.0 \mathrm{mmol})$, ethyl glyoxylate $(1.02 \mathrm{~mL}, 5.00$ $\mathrm{mmol}, 50 \%$ in toluene) and pyrrole $(1.39 \mathrm{~mL}, 20.0 \mathrm{mmol})$ in $\mathrm{CHCl}_{3}(274 \mathrm{~mL})$ was treated with $\mathrm{BF}_{3} \cdot \mathrm{O}(\mathrm{Et})_{2}(0.618 \mathrm{~mL}, 4.88 \mathrm{mmol})$ under argon at room temperature. After $20 \mathrm{~min}$, DIEA $(0.850 \mathrm{~mL}, 4.88 \mathrm{mmol})$ was added. DDQ $(3.41 \mathrm{~g}, 15.0 \mathrm{mmol})$ was added and the reaction mixture was stirred at room temperature for $1 \mathrm{~h}$. The mixture was filtered through a silica pad $\left(\mathrm{CH}_{2} \mathrm{Cl}_{2}\right)$. The purple fraction was collected, concentrated and chromatographed on silica, affording a purple solid $(106 \mathrm{mg}, 3 \%):{ }^{1} \mathrm{H}$ NMR $\delta-2.62(\mathrm{~s}, 2 \mathrm{H}), 1.84-1.86(\mathrm{~m}, 21 \mathrm{H}), 2.62(\mathrm{~s}$, $3 \mathrm{H}), 2.64$ (s, 6H), 5.07 (q, $J=7.2 \mathrm{~Hz}, 2 \mathrm{H}), 7.30(\mathrm{~s}, 2 \mathrm{H}), 7.32(\mathrm{~s}, 2 \mathrm{H}), 7.33(\mathrm{~s}, 2 \mathrm{H}), 8.62$ (d, $J=$ $4.4 \mathrm{~Hz}, 2 \mathrm{H}), 8.65$ (d, $J=4.8 \mathrm{~Hz}, 2 \mathrm{H}), 8.79(\mathrm{~d}, J=4.8 \mathrm{~Hz}, 2 \mathrm{H}), 9.38$ (d, $J=4.8 \mathrm{~Hz}, 2 \mathrm{H})$; LD-MS obsd 737.8; FAB-MS obsd 736.3810, calcd $736.3777\left(\mathrm{C}_{50} \mathrm{H}_{48} \mathrm{~N}_{4} \mathrm{O}_{2}\right) ; \lambda_{\text {abs }} 419,513,546,589,645$ nm.

5-Ethoxycarbonyl-10,15,20-trimesitylporphinatozinc(II) (Zn-1). A solution of 1 (100 $\mathrm{mg}, 0.136 \mathrm{mmol})$ in $\mathrm{CHCl}_{3}(15 \mathrm{~mL})$ was treated with a solution of $\mathrm{Zn}(\mathrm{OAc})_{2} \cdot 2 \mathrm{H}_{2} \mathrm{O}(298 \mathrm{mg}$, $1.36 \mathrm{mmol})$ in methanol $(3 \mathrm{~mL})$ at room temperature. The reaction was monitored by fluorescence spectroscopy. After the metalation was complete, the mixture was washed with water. The organic phase was dried $\left(\mathrm{Na}_{2} \mathrm{SO}_{4}\right)$, concentrated and chromatographed [silica, $\mathrm{CHCl}_{3}$ $\rightarrow \mathrm{CHCl}_{3}$ /ethyl acetate (95:5)], affording a purple solid (100 mg, 92\%): ${ }^{1} \mathrm{H}$ NMR $\delta 1.84-1.86$ $(\mathrm{m}, 21 \mathrm{H}), 2.60(\mathrm{~s}, 3 \mathrm{H}), 2.63(\mathrm{~s}, 6 \mathrm{H}), 5.02(\mathrm{q}, J=7.2 \mathrm{~Hz}, 2 \mathrm{H}), 7.29(\mathrm{~s}, 2 \mathrm{H}), 7.32(\mathrm{~s}, 4 \mathrm{H}), 8.60$ (d, $J=4.2 \mathrm{~Hz}, 2 \mathrm{H}), 8.63$ (d, $J=4.8 \mathrm{~Hz}, 2 \mathrm{H}), 8.72$ (d, $J=4.8 \mathrm{~Hz}, 2 \mathrm{H}), 9.38$ (d, $J=4.5 \mathrm{~Hz}, 2 \mathrm{H})$; LD- 
MS obsd 799.8; FAB-MS obsd 799.2997, calcd $799.2990\left[(\mathrm{M}+\mathrm{H})^{+} ; \mathrm{M}=\mathrm{C}_{50} \mathrm{H}_{46} \mathrm{~N}_{4} \mathrm{O}_{2} \mathrm{Zn}\right] ; \lambda_{\text {abs }}$ $422,551,588 \mathrm{~nm}$.

5-Hydroxymethyl-10,15,20-trimesitylporphinatozinc(II) $\quad$ (mes- $\left.\mathrm{ZnPCH}_{2} \mathrm{OH}\right) . \quad$ A solution of $\mathbf{Z n - 1}(95.0 \mathrm{mg}, 119 \mu \mathrm{mol})$ in dry THF $(8 \mathrm{~mL})$ was treated with $\mathrm{LiAlH}_{4}(14.0 \mathrm{mg}, 356$ $\mu \mathrm{mol})$ and the reaction mixture was stirred at room temperature. After the reaction was completed, methanol $(5 \mathrm{~mL})$ was slowly added and the mixture was filtered to remove insoluble compound. After removal of the solvent, the crude product was purified by column chromatography on silica, affording a purple solid $(31 \mathrm{mg}, 34 \%): 1.84-1.86(\mathrm{~m}, 18 \mathrm{H}), 2.53(\mathrm{~s}$, $6 \mathrm{H}), 2.60(\mathrm{~s}, 2 \mathrm{H}), 2.63(\mathrm{~s}, 3 \mathrm{H}), 6.89(\mathrm{~d}, J=6.0 \mathrm{~Hz}, 2 \mathrm{H}), 7.28(\mathrm{~s}, 2 \mathrm{H}), 7.31(\mathrm{~s}, 4 \mathrm{H}), 8.60(\mathrm{~s}, 2 \mathrm{H})$, $8.73(\mathrm{~d}, J=4.8 \mathrm{~Hz}, 2 \mathrm{H}), 8.69(\mathrm{~d}, J=4.4 \mathrm{~Hz}, 2 \mathrm{H})$; LD-MS obsd 740.8 [(M - OH) $\left.{ }^{+}\right], 758.0$; FABMS obsd 756.28437, calcd 756.2807 $\left(\mathrm{C}_{48} \mathrm{H}_{44} \mathrm{~N}_{4} \mathrm{OZn}\right) ; \lambda_{\text {abs }} 421,550,582 \mathrm{~nm}$.

\section{References.}

(S1) Lindsey, J. S.; Wagner, R. W. J. Org. Chem. 1989, 54, 828-836.

(S2) (a) Srinivasan, N.; Haney, C. A.; Lindsey, J. S.; Zhang, W.; Chait, B. T. J. Porphyrins Phthalocyanines 1999, 3, 283-291. (b) Fenyo, D.; Chait, B. T.; Johnson, T. E.; Lindsey, J. S. J. Porphyrins Phthalocyanines 1997, 1, 93-99. 


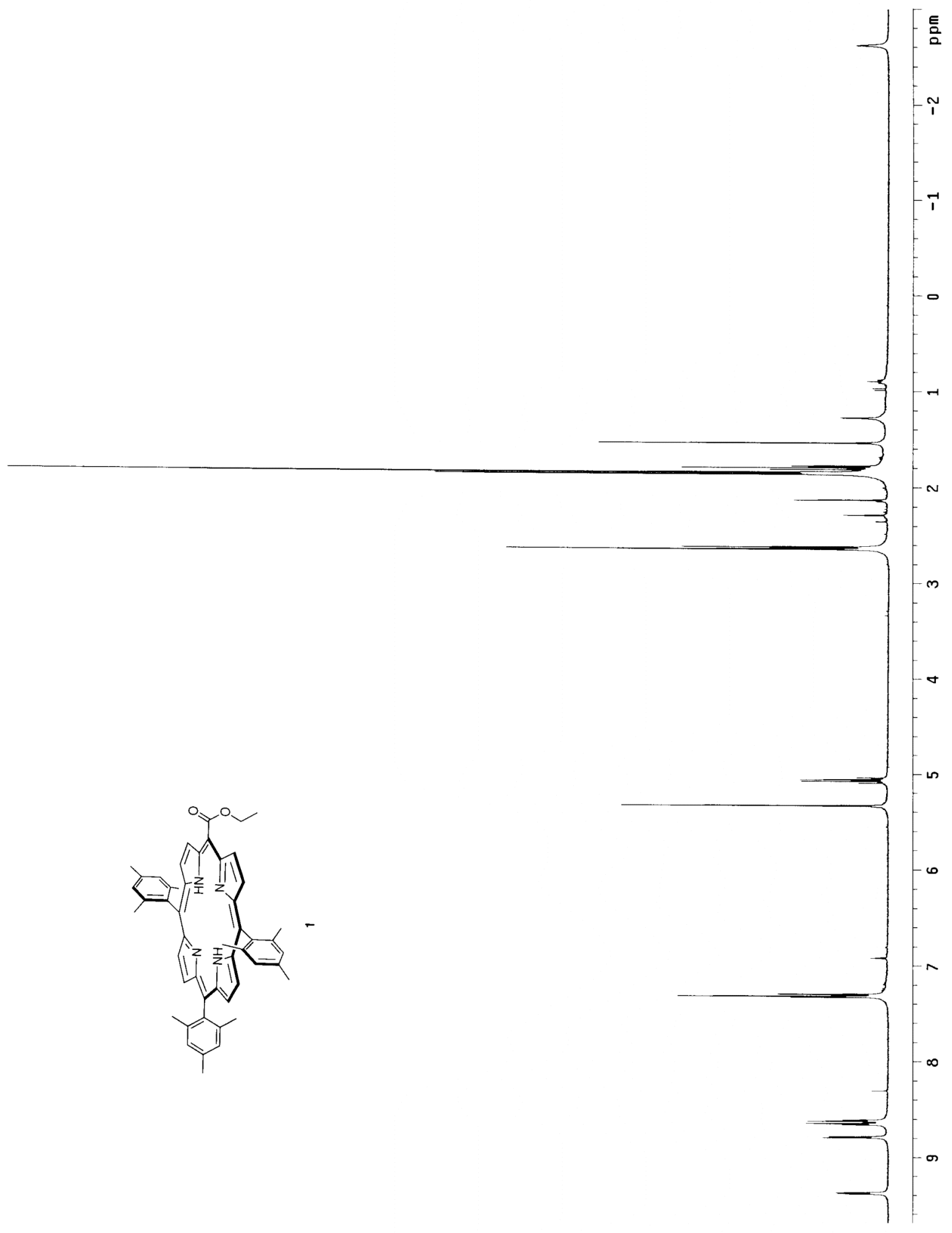




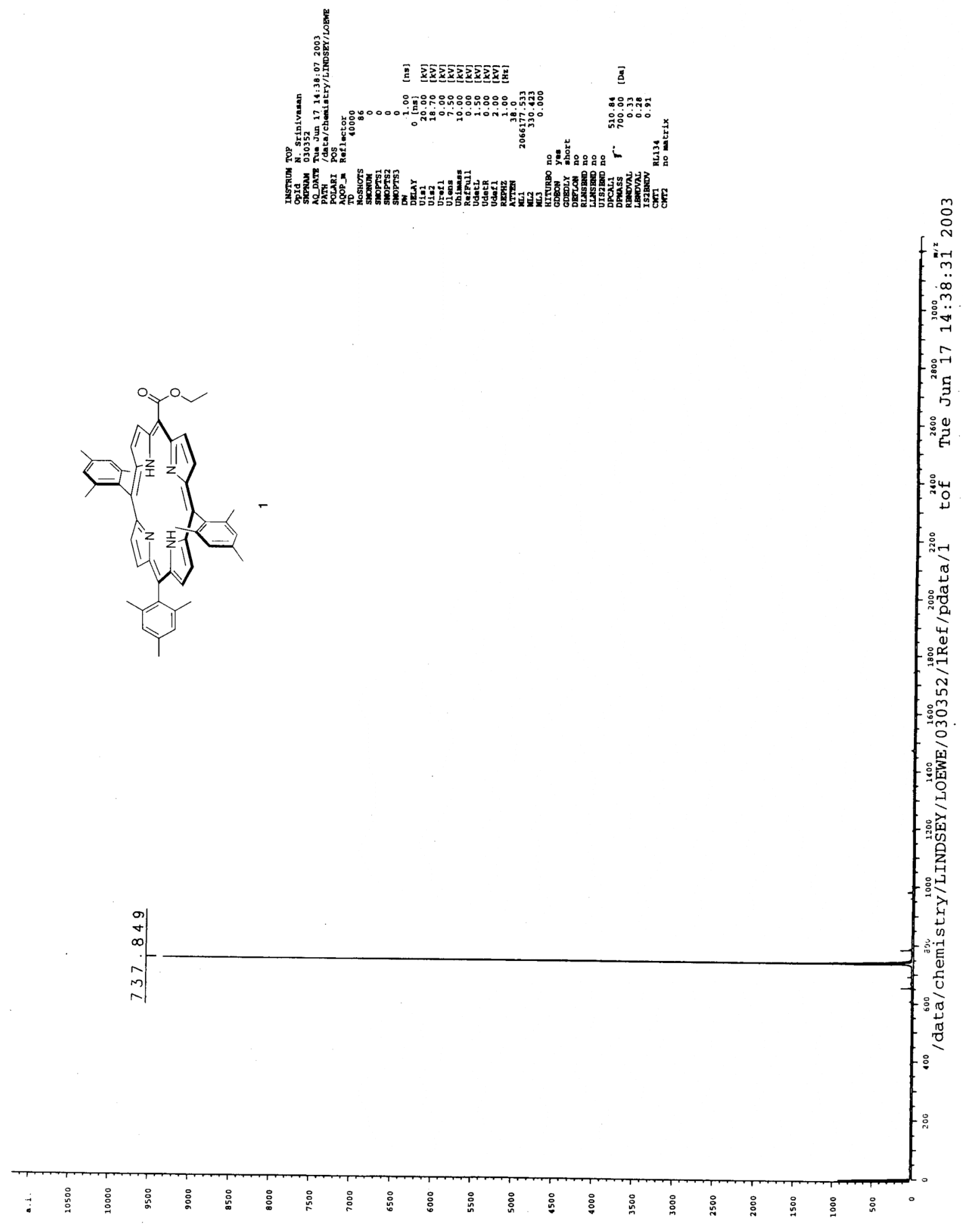




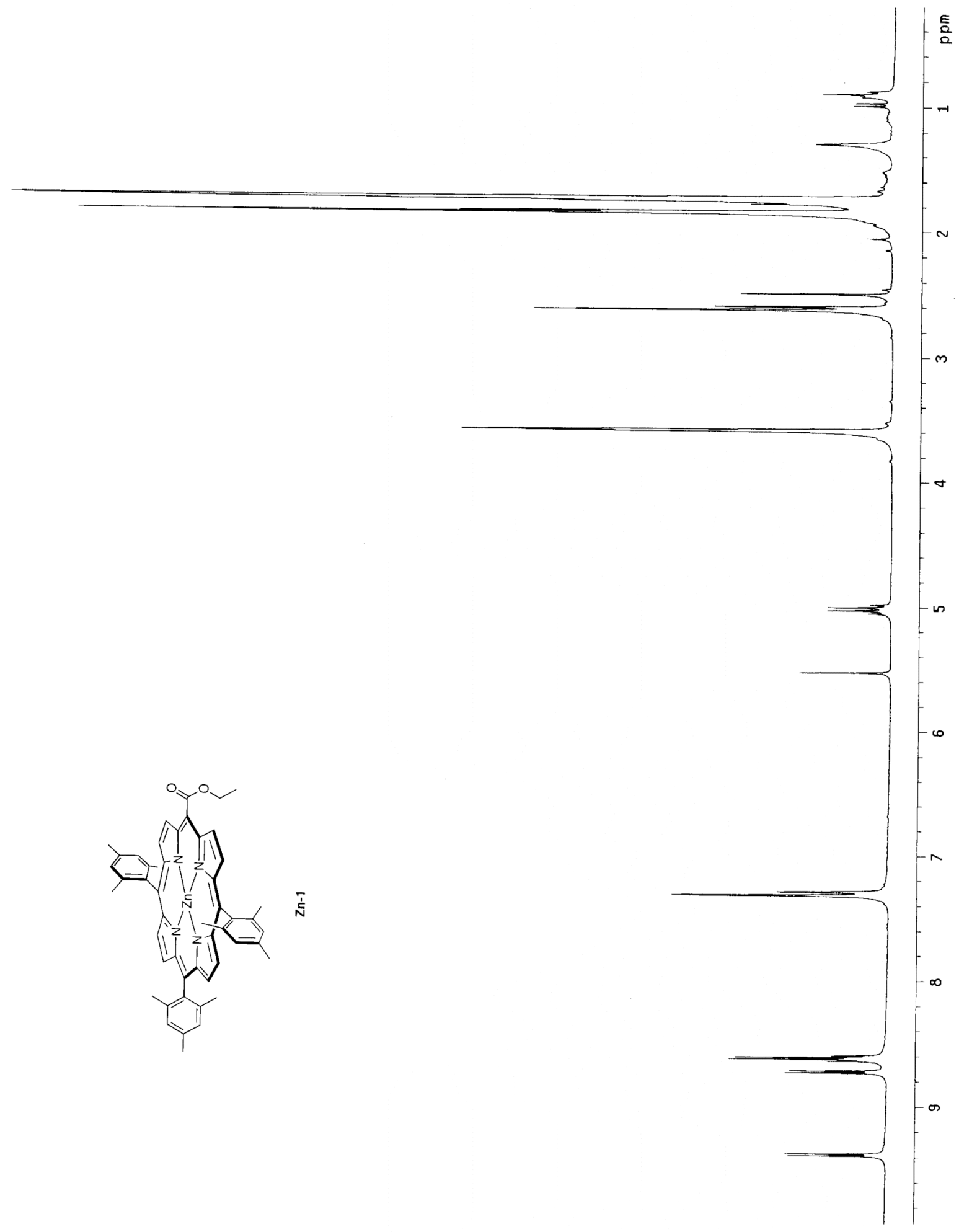




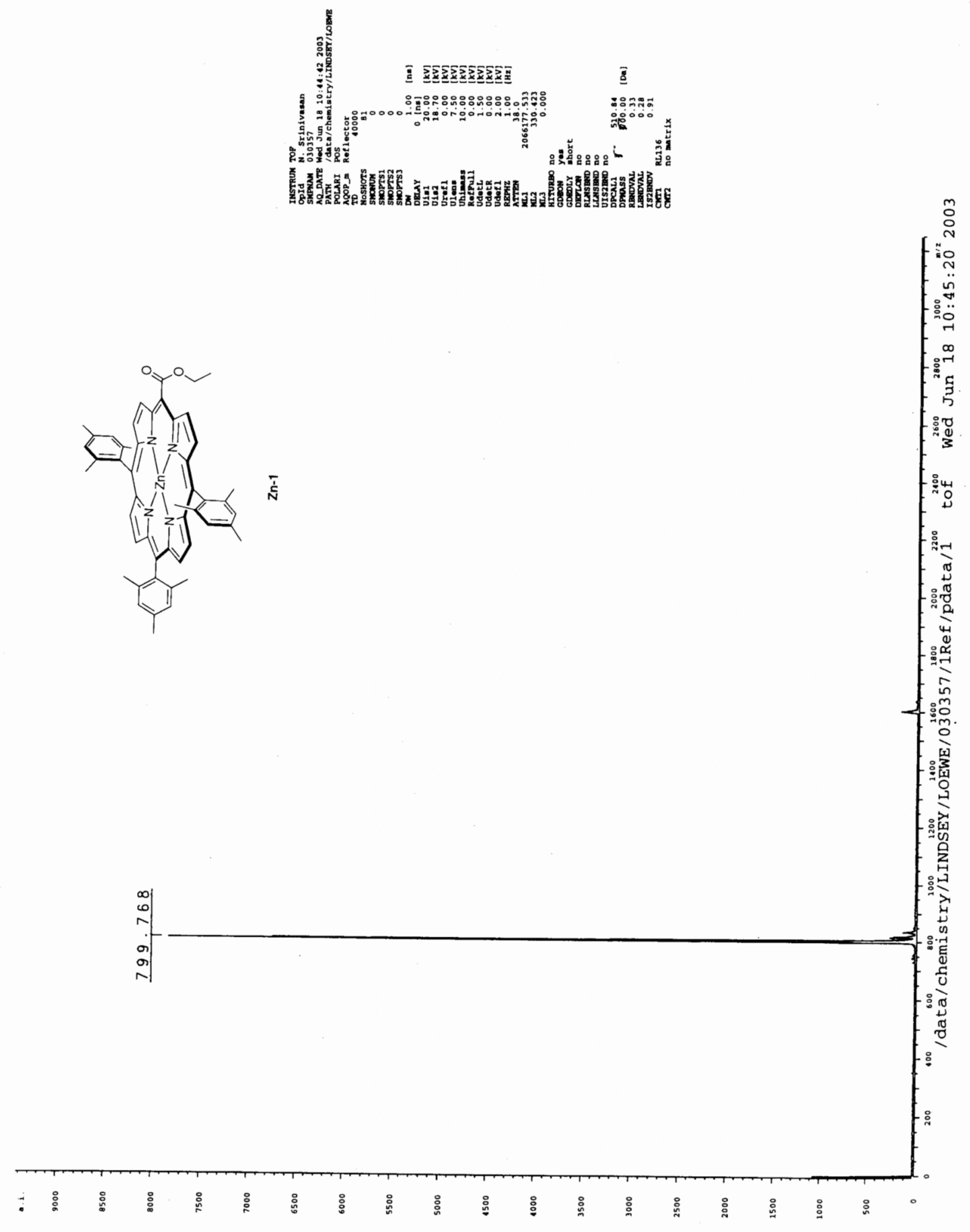




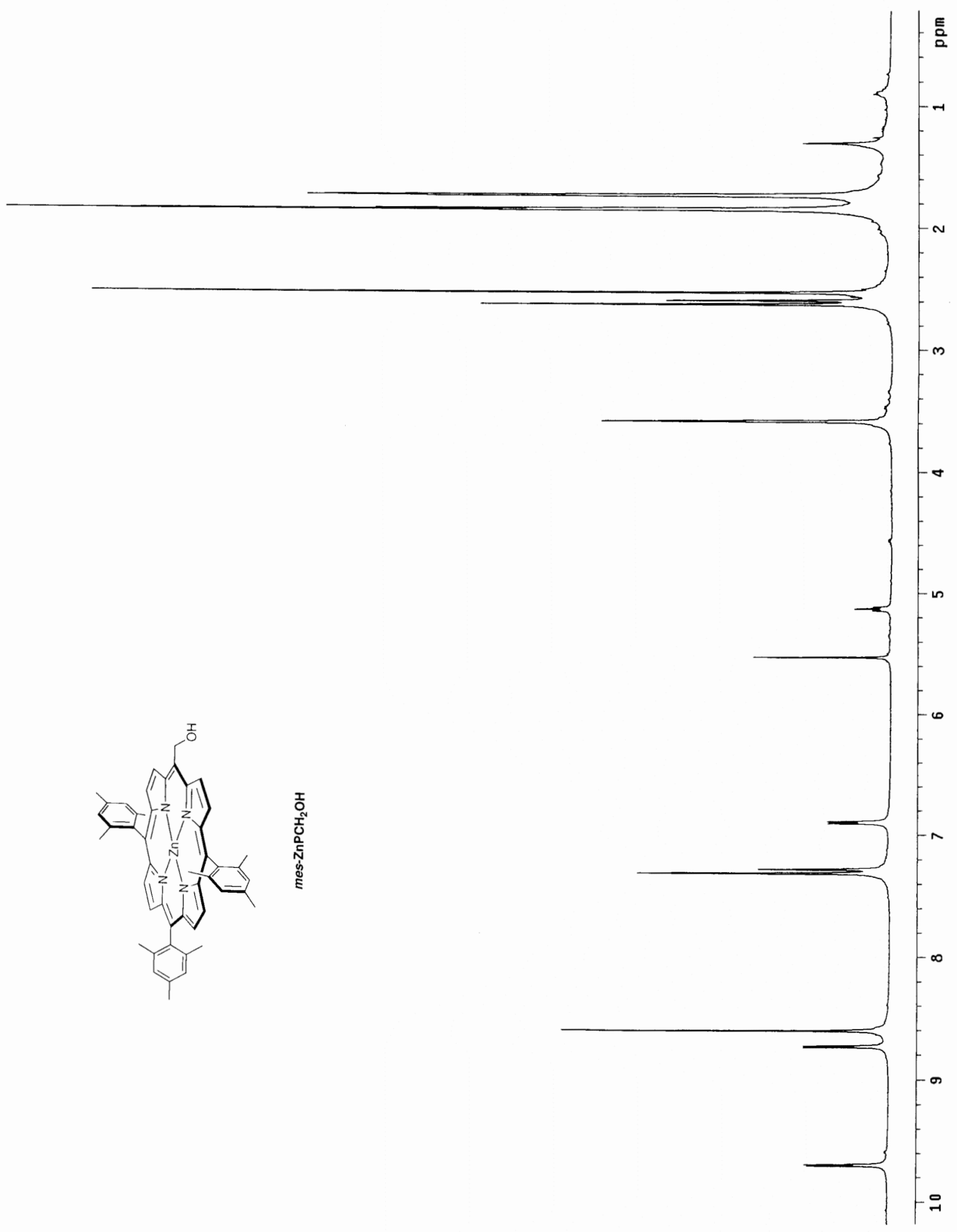




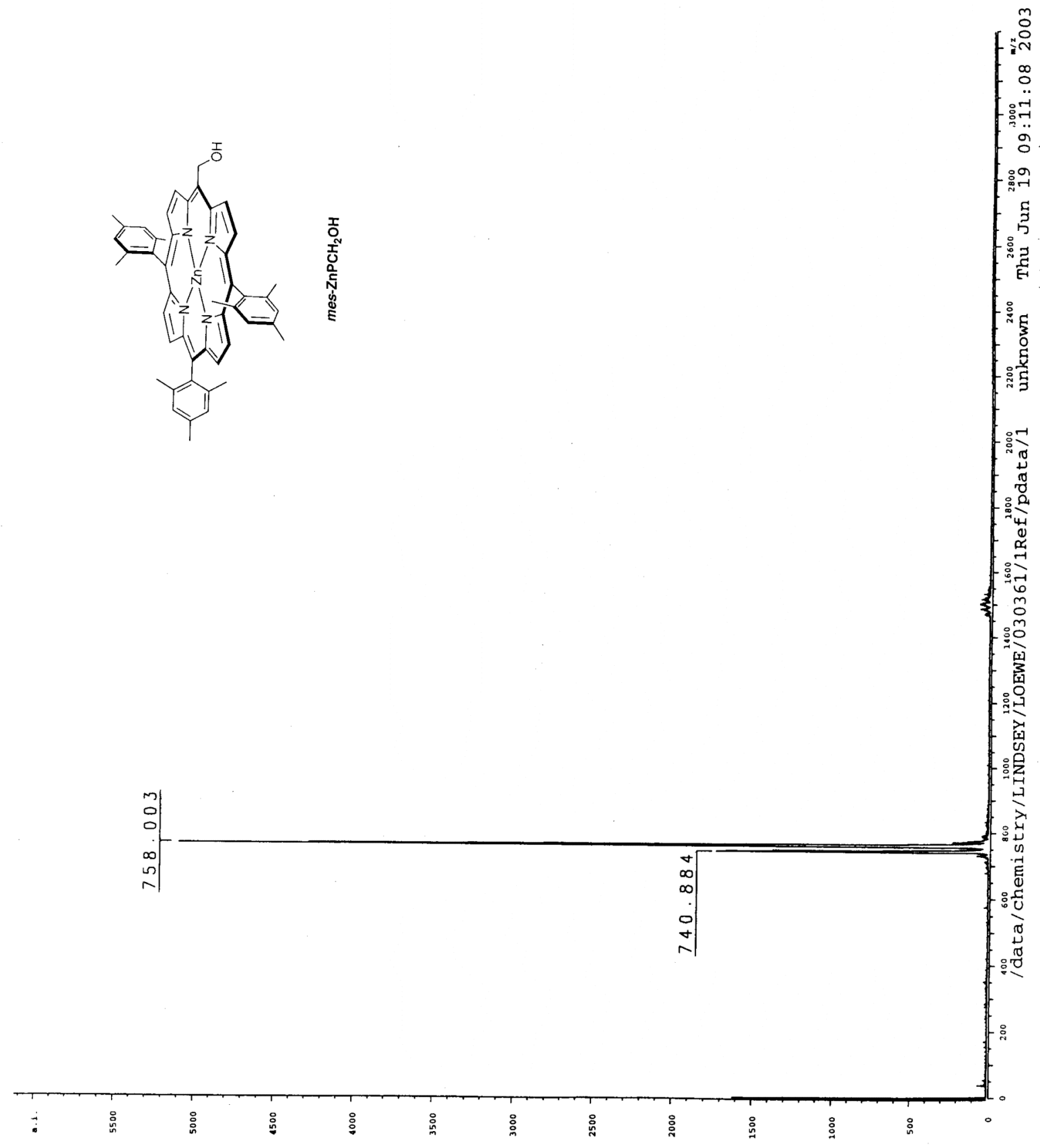

